\title{
STRUCTURAL INFERENCE WITH LONG-RUN RECURSIVE EMPIRICAL MODELS
}

\author{
JoHn W. KeAting \\ University of Kansas
}

This paper investigates conditions under which a long-run recursive model can be used to identify a structure. Economists frequently employ this type of empirical model. I define the class of long-run partially recursive structures. If an economic system is a member of this class, then certain long-run recursive empirical models will obtain some of the structural impulse response functions. This sufficient condition for a structure is first shown in a vector autoregression. A well-known example from the literature is used to illustrate this particular class of structures and to present some useful applications of the result. Then the result is shown in models of cointegrated time series. Necessary conditions for a long-run recursive model to identify structure are addressed in the conclusion.

Keywords: Long-Run Multiplier, Long-Run Partially Recursive Structure, Moving Average Representation, Vector Autoregression, Cointegration

\section{INTRODUCTION}

Recursive models have a long history in empirical macroeconomic research. ${ }^{1}$ More recently Sims (1980) began using recursive models to identify vector autoregressions (VAR's). A practical benefit of recursive models is that, in general, parameters are identified. In practice, the coefficients in Sims's short-run recursive models are obtained easily by a Cholesky decomposition of the covariance matrix for VAR innovations. However, Cooley and LeRoy (1985) and others have criticized Sims's approach for being "atheoretical." In response to this criticism, economists have devised various structural approaches to VAR modeling.

One such approach identifies economic structure using long-run restrictions derived from the steady-state properties of a theoretical model. This method is particularly attractive to economists who believe that economic theory describes longrun equilibrium behavior better than short-run dynamics. Following Blanchard and Quah (1989), empirical work based on long-run restrictions often uses recursive identifying assumptions. A purely recursive long-run multiplier matrix is estimated easily by means of a Cholesky decomposition of a matrix constructed from the

I thank Mark Dwyer, Ed Greenberg, Mark Watson, the referees, an Associate Editor, and seminar participants at UCLA, the University of Kansas, the Midwest Econometrics Group Meeting and the Midwest Macroeconomics Conference for beneficial comments. Any errors would of course be attributable to me. Address correspondence to: John W. Keating, Associate Professor, University of Kansas, Department of Economics, 213 Summerfield Hall, Lawrence, KS 66045, USA; e-mail: jkeating@ku.edu. 
covariance matrix for VAR innovations and the sum of VAR coefficients. However, the set of recursive systems is quite limited compared to the set of all possible economic structures. Therefore, some economists might be concerned that the criticisms leveled against short-run recursive orderings may also apply to long-run recursive orderings. ${ }^{2}$

A number of potential pitfalls with long-run identification restrictions have been addressed in the literature, starting with the appendix to Blanchard and Quah (1989). Faust and Leeper (1997) extend Blanchard and Quah's investigation in some important and interesting directions. ${ }^{3}$ Other studies deal with potential econometric difficulties. Models that use long-run identification assumptions often can be estimated by means of an instrumental variables method developed by Shapiro and Watson (1988) in which residuals from the structural equations serve as instruments. Pagan and Robertson (1998) and Sarte (1997) examine inference problems that may arise if residuals are nearly uncorrelated with the variables that require instruments. $^{4}$

This paper also is concerned with the usefulness of empirical models that employ long-run identification restrictions. However, in contrast to other research, I investigate general conditions under which long-run recursive empirical models are able to identify structural relationships. The frequent use of long-run recursive models in empirical research is a primary motivation for this study. If an economic structure is long-run block recursive, if the structural equations in at least one of these blocks can be recursively ordered, and if the structural shocks are uncorrelated, I show that certain long-run recursive empirical models will obtain some structural impulse responses. Economies that satisfy these three conditions are called long-run partially recursive structures. If the chosen long-run ordering is consistent with this structure, the empirical model will yield structural responses to each shock from the particular block of long-run recursive structural equations. All other shocks from this empirical model will not identify structural effects. The finding that the block of long-run recursive equations can occur anywhere within a block-recursive structure has not been shown before. ${ }^{5}$

The paper is composed as follows. Section 2 describes a popular method for constructing long-run recursive orderings in VAR's with differenced data and shows the relationship between a long-run recursive ordering and a general economic structure. Section 3 uses linear projection arguments to prove that a long-run partially recursive structure will permit long-run recursive orderings to identify some structural responses in a VAR model with differenced data. Section 4 presents examples of long-run partially recursive structures, based on the economic theory developed by Amed et al. (1993), to illustrate this class of structures and to clarify some of the ways that the result from Section 3 may be used. Section 5 extends the result to models with cointegrated data. Hence, the sufficient condition for a structure to be identifiable from long-run recursive identification restrictions applies to some of the most popular multivariate time series models used in economics. Section 6 concludes the paper and briefly discusses necessary conditions for a long-run recursive model to identify structure. 


\section{IMPLEMENTING LONG-RUN RECURSIVE ORDERINGS}

This section examines recursive orderings under general structural assumptions. I show that when the economy's long-run structure is not recursive, a long-run recursive empirical model will not, in general, yield structural impulse response functions. Presenting this well-known result is a useful starting point for determining conditions that will permit long-run recursive orderings to produce structural responses.

The structural moving average representation (MAR) is a convenient tool for studying economic systems that are usually written in autoregressive form. This representation writes each endogenous variable as a function of current and past structural shocks. If $\boldsymbol{y}_{t}$ is an $n$-vector of difference-stationary time series and $\boldsymbol{\epsilon}_{t}$ is the vector of $n$ structural shocks, the structural MAR is

$$
\Delta \boldsymbol{y}_{t}=\theta(L) \boldsymbol{\epsilon}_{t},
$$

where $\theta(L)=\sum_{j=0}^{\infty} \theta_{j} L^{j}$ and $\theta_{j}$ is an $n \times n$ matrix of parameters for $j=$ $0,1,2, \ldots, \infty$. Deterministic elements can be omitted without loss of generality. ${ }^{6}$ An economist would like the VAR model's impulse responses to uncover equation (1).

The multivariate Beveridge and Nelson (1981) decomposition for $\boldsymbol{y}_{t}$ is ${ }^{7}$

$$
\boldsymbol{y}_{t}=\overline{\boldsymbol{y}}+\theta(1) \sum_{j=0}^{\infty} \boldsymbol{\epsilon}_{t-j}+\theta^{*}(L) \boldsymbol{\epsilon}_{t},
$$

where

$$
\theta^{*}(L)=\frac{\theta(L)-\theta(1)}{1-L}=\sum_{i=0}^{\infty} \theta_{i}^{*} L^{i}
$$

with

$$
\theta_{i}^{*}=-\sum_{k=i+1}^{\infty} \theta_{k}
$$

and $\bar{y}$ is the initial condition for $\boldsymbol{y}$. If $\Delta \boldsymbol{y}_{t}$ is stationary, then the last term in equation (2) is a stationary multivariate moving average process. Consequently, this term has no effect on the level of $\boldsymbol{y}$ in the long run. The second term in (2) sums the vector of structural shocks, indicating that each shock potentially may have a permanent effect on $\boldsymbol{y}$. The magnitude and direction of these permanent effects is given by the matrix of long-run structural multipliers, $\theta(1)$, which is the sum of structural parameters in $\theta(L)$.

The VAR representation is given by

$$
b(L) \Delta \boldsymbol{y}_{t}=\boldsymbol{v}_{t},
$$


where $v_{t}$ is an $n$-vector of VAR innovations and $b(L)=I-b_{1} L-b_{2} L^{2}-\cdots$ $-b_{\ell} L^{\ell}$ with $b_{j}$ the $n \times n$ matrix of coefficients on variables lagged $j$ periods and $I$ the $n \times n$ identity matrix. The VAR representation is derived from the underlying structural MAR by premultiplying (1) by the inverse ${ }^{8}$ of $\theta(L)$ and then premultiplying the resulting expression by $\theta(0)$. Consequently, the VAR's coefficients are a function of the parameters in the structural MAR, ${ }^{9}$

$$
b(L)=\theta(0) \theta(L)^{-1}
$$

and the VAR innovations are given by

$$
\boldsymbol{v}_{t}=\theta(0) \epsilon_{t} .
$$

The structural shocks are generally assumed to be uncorrelated white noise processes. Thus, equation (5) shows that each innovation is also serially uncorrelated, because it is a linear combination of white noise structural shocks, and that these linear combinations are based on the short-run structural parameters, $\theta(0)$. The diagonal covariance matrix for $\epsilon_{t}$ is conveniently normalized to be an identity. Therefore, if $\Sigma_{v}$ is the covariance matrix for VAR innovations, equation (5) implies that

$$
\Sigma_{v}=\theta(0) \theta(0)^{\prime}
$$

Economists who favor short-run identification restrictions make use of this relationship. For example, Sims (1980) used Cholesky decompositions of $\Sigma_{v}$ for identification purposes. Given a particular ordering of the residuals, a Cholesky decomposition can be obtained by finding the unique ${ }^{10}$ lower triangular matrix $C$ that solves

$$
\Sigma_{v}=C C^{\prime}
$$

The first generation of structural VAR models applied restrictions on $\theta(0)$ that were derived from economic theory. Clearly, if $\theta(0)$ is lower triangular, then $C=\theta(0)$ because the recursive factor is unique. Methods other than the Cholesky decomposition are required, however, if one wishes to identify all of the parameters for an economic structure that is not recursive. ${ }^{11}$

In contrast, structures derived from economic assumptions about long-run or steady-state behavior impose restrictions on the matrix of long-run multipliers. A relationship between long-run structural multipliers, short-run structural parameters, and the sum of VAR coefficients, $b(1)$, is obtained by letting $L=1$ in equation (4):

$$
b(1)=\theta(0) \theta(1)^{-1}
$$

One can solve (8) for $\theta(0)$, insert this result into (6), and simplify to obtain

$$
b(1)^{-1} \Sigma_{v}\left[b(1)^{-1}\right]^{\prime}=\theta(1) \theta(1)^{\prime} .
$$


This equation maps the long-run structural parameters into parameters from the reduced form.

A long-run recursive ordering is obtained by finding the representation

$$
\Delta \boldsymbol{y}_{t}=R(L) \boldsymbol{u}_{t},
$$

such that the matrix of long-run multipliers for $\boldsymbol{u}_{t}$, given by $R(1)$, is triangular. The assumption that the $\boldsymbol{u}_{t}$ shocks are contemporaneously uncorrelated yields a diagonal covariance matrix, which, for convenience, is normalized to be the identity matrix. The MAR in (10) is mapped into the VAR representation following the same procedure that was used with the structural system, yielding

$$
b(L)=R(0) R(L)^{-1}
$$

and

$$
\boldsymbol{v}_{t}=R(0) \boldsymbol{u}_{t}
$$

To construct $R(1)$ from the VAR, first set $L=1$ in (11):

$$
b(1)=R(0) R(1)^{-1}
$$

and use (12) to find

$$
\Sigma_{v}=R(0) R(0)^{\prime}
$$

Then, solve (13) for $R(0)$, eliminate $R(0)$ from (14), and simplify to obtain

$$
b(1)^{-1} \Sigma_{v}\left[b(1)^{-1}\right]^{\prime}=R(1) R(1)^{\prime} .
$$

A convenient way to calculate $R(1)$ is by a Cholesky decomposition of the left side of equation (15).

Coefficients from the long-run recursive model can be related to the structural parameters using (9) and (15):

$$
R(1) R(1)^{\prime}=\theta(1) \theta(1)^{\prime} .
$$

In the most general case, $\theta(1)$ is not lower triangular, and therefore each coefficient in $R(1)$ is a nonlinear function of the $\theta(1)$ structural parameters. However, if the economic structure is recursive in the long run, that is, if the variables can be ordered so that $\theta(1)$ is lower triangular, then

$$
R(1)=\theta(1)
$$

because the triangular factor matrix is unique. In other words, if the structural system is long-run recursive and the economist chooses the correct ordering, the matrix of long-run structural multipliers is identified.

The relationship between the structural MAR in (1) and the MAR obtained by the long-run recursive model in (10) is of primary interest. Empirical researchers 
construct the MAR in (10) by premultiplying (3) by $b(L)^{-1}$ and by using (12) to eliminate VAR innovations:

$$
\Delta \boldsymbol{y}_{t}=b(L)^{-1} \boldsymbol{v}_{t}=b(L)^{-1} R(0) \boldsymbol{u}_{t} .
$$

To relate $R(L)$ to $\theta(L)$, use (4) to eliminate $b(L)$ in (18):

$$
\Delta \boldsymbol{y}_{t}=\theta(L) \theta(0)^{-1} R(0) \boldsymbol{u}_{t}=R(L) \boldsymbol{u}_{t} .
$$

Obviously, if $R(0)=\theta(0)$, then $R(L)=\theta(L)$. But, given that the paper is concerned with long-run identification restrictions, equation (19) should instead be put in terms of long-run structural parameters and coefficients from the long-run recursive model. Solving (8) for $\theta(0)$ and (13) for $R(0)$ yields

$$
\theta(0)^{-1} R(0)=\theta(1)^{-1} b(1)^{-1} b(1) R(1)=\theta(1)^{-1} R(1) .
$$

Substituting this result from (20) into (19) gives

$$
R(L)=\theta(L) \theta(1)^{-1} R(1) .
$$

If the long-run structure is recursive and the economist selects the correct recursive ordering, then (17) obtains, and plugging (17) into (21) yields $R(L)=\theta(L)$. In other words, the long-run recursive empirical model will identify all of the structural responses if the economy has a long-run recursive structure and the correct recursive ordering is selected. However, if the structure is not long-run recursive, then the MAR associated with $\boldsymbol{u}_{t}$ will generally be a complicated function of the structural dynamics.

\section{STRUCTURAL INFERENCE USING VAR MODELS WITH DIFFERENCED DATA}

The objective of this paper is to determine general conditions under which a longrun recursive ordering is able to identify a structure. First, I define the class of long-run partially recursive structures. Then I show that if the economic structure is a member of this class, certain long-run recursive orderings can be applied to a VAR with differenced data to obtain some structural impulse response functions.

DEFINITION 1. A long-run partially recursive structure consists of

(i) a structural system with a block-recursive matrix of long-run multipliers;

(ii) equations in one of these structural blocks that can be ordered recursively;

(iii) structural shocks that are uncorrelated.

The system from Section 2 is modified to consider a long-run partially recursive structure. Let the vector of $n$ variables in the structural system be broken into three groups: The first $n_{1}$ variables are in $\boldsymbol{y}_{1 t}$, the next $n_{2}$ variables are in $\boldsymbol{y}_{2 t}$, the final $n_{3}$ variables are in $\boldsymbol{y}_{3 t}$ and $n_{1}+n_{2}+n_{3}=n$. Let the vector of structural shocks be similarly partitioned so that $\epsilon_{1 t}$ has the first $n_{1}$ shocks, $\epsilon_{2 t}$ holds the next $n_{2}$ 
shocks, and $\epsilon_{3 t}$ contains the final $n_{3}$ shocks. Once again, the covariance matrix for uncorrelated structural shocks is normalized to be the identity matrix. ${ }^{12}$ Hence, the structural MAR is

$$
\left[\begin{array}{l}
\Delta \boldsymbol{y}_{1 t} \\
\Delta \boldsymbol{y}_{2 t} \\
\Delta \boldsymbol{y}_{3 t}
\end{array}\right]=\left[\begin{array}{lll}
\theta_{11}(L) & \theta_{12}(L) & \theta_{13}(L) \\
\theta_{21}(L) & \theta_{22}(L) & \theta_{23}(L) \\
\theta_{31}(L) & \theta_{32}(L) & \theta_{33}(L)
\end{array}\right]\left[\begin{array}{l}
\boldsymbol{\epsilon}_{1 t} \\
\boldsymbol{\epsilon}_{2 t} \\
\boldsymbol{\epsilon}_{3 t}
\end{array}\right],
$$

where $\theta_{i j}(L)=\theta_{i j 0}+\theta_{i j 1} L+\theta_{i j 2} L^{2}+\ldots$, with $\theta_{i j k}$ an $n_{i} \times n_{j}$ matrix for all nonnegative integer $k, i=1,2,3$ and $j=1,2,3$. The long-run multiplier matrix for shocks from equation (22), in general, is written as

$$
\theta(1)=\left[\begin{array}{lll}
\theta_{11} & \theta_{12} & \theta_{13} \\
\theta_{21} & \theta_{22} & \theta_{23} \\
\theta_{31} & \theta_{32} & \theta_{33}
\end{array}\right]
$$

where I define $\theta_{i j}=\theta_{i j}(1)$.

Assumption 1. The structure is long-run partially recursive from the following restrictions:

$\theta_{12}=0_{12}, \theta_{13}=0_{13}, \theta_{23}=0_{23}$, where $0_{i j}$ is an $n_{i} \times n_{j}$ matrix of zeros; that is,

$$
\theta(1)=\left[\begin{array}{lll}
\theta_{11} & 0_{12} & 0_{13} \\
\theta_{21} & \theta_{22} & 0_{23} \\
\theta_{31} & \theta_{32} & \theta_{33}
\end{array}\right]
$$

and $\theta_{22}$ is a lower triangular $n_{2} \times n_{2}$ matrix.

Placing the block of recursive equations in the interior of this block-recursive system yields a general form of the long-run partially recursive structure. In Section 3 , I discuss some interesting special cases of this general form. Except for $\theta_{22}$, all nonzero parameter matrices in equation (24) are unconstrained. If both $\theta_{11}$ and $\theta_{33}$ were also lower triangular, the analysis in Section 2 shows that the appropriate long-run recursive ordering would identify dynamic responses for each structural shock.

PROPOSITION 1. If an economic structure is long-run partially recursive, each structural shock has a permanent effect on at least one of the variables and the data are not cointegrated, then a VAR with differenced data is able to recover some structural impulse responses with long-run recursive identifying restrictions, as long as the recursive model is consistent with the underlying structure.

A proof of Proposition 1 begins by considering the following linear combinations of the $\epsilon_{1 t}$ structural disturbances:

$$
\lambda_{1 t}=\theta_{11} \epsilon_{1 t}
$$


where each linear combination is written as

$$
\lambda_{1 t}^{i}=\sum_{j=1}^{n_{1}} \theta_{11}^{i j} \epsilon_{1 t}^{j} \quad \text { for } \quad i=1,2, \ldots, n_{1}
$$

with superscripts indicating particular elements in $\epsilon_{1 t}, \lambda_{1 t}$, and $\theta_{11}$. Linear projection equations with $\lambda_{1 t}$ are used to map this structure into the long-run recursive empirical model. First, project the second element in $\boldsymbol{\lambda}_{1 t}$ onto the first element:

$$
\lambda_{1 t}^{2}=P_{1}^{21} \lambda_{1 t}^{1}+\rho_{1 t}^{2},
$$

where $P_{1}^{21}$ is the projection coefficient and $\rho_{1 t}^{2}$ is the projection error. Continue projecting each variable in $\boldsymbol{\lambda}_{1 t}$ onto all preceding variables:

$$
\begin{aligned}
\lambda_{1 t}^{3} & =P_{1}^{31} \lambda_{1 t}^{1}+P_{1}^{32} \lambda_{1 t}^{2}+\rho_{1 t}^{3} \\
& \vdots \\
\lambda_{1 t}^{n_{1}} & =P_{1}^{n_{1} 1} \lambda_{1 t}^{1}+\cdots+P_{1}^{n_{1}, n_{1}-1} \lambda_{1 t}^{n_{1}-1}+\rho_{1 t}^{n_{1}},
\end{aligned}
$$

where projection errors are indexed by the dependent variable and projection coefficients are indexed by the dependent variable and the explanatory variable, respectively. Along with the identity that sets $\lambda_{1 t}^{1}$ equal to itself, this system of projection equations can be written as

$$
\left[\begin{array}{ccccc}
1 & 0 & 0 & \cdot & 0 \\
-P_{1}^{21} & 1 & 0 & . & 0 \\
-P_{1}^{31} & -P_{1}^{32} & 1 & \cdot & 0 \\
\cdot & \cdot & \cdot & \cdot & \cdot \\
-P_{1}^{n_{1} 1} & \cdot & \cdot & -P_{1}^{n_{1}, n_{1}-1} & 1
\end{array}\right]\left[\begin{array}{c}
\lambda_{1 t}^{1} \\
\lambda_{1 t}^{2} \\
\lambda_{1 t}^{3} \\
\cdot \\
\lambda_{1 t}^{n_{1}}
\end{array}\right]=\left[\begin{array}{c}
\lambda_{1 t}^{1} \\
\rho_{1 t}^{2} \\
\rho_{1 t}^{3} \\
\cdot \\
\rho_{1 t}^{n_{1}}
\end{array}\right]
$$

or more conveniently as

$$
P_{1} \lambda_{1 t}=\rho_{1 t}
$$

where $P_{1}$ is the lower triangular matrix of projection coefficients and $\rho_{1 t}$ is the vector of projection errors. The covariance matrix for $\rho_{1 t}$ is given by $E \rho_{1 t} \rho_{1 t}^{\prime}=D_{1}$, where $D_{1}$ is a diagonal matrix by construction. Using $D_{1}^{1 / 2}$ as the square root of the diagonal covariance matrix, the vector of projection errors can be written as

$$
\rho_{1 t}=D_{1}^{1 / 2} u_{1 t}
$$

where $\boldsymbol{u}_{1 t}$ has an identity covariance matrix, $E \boldsymbol{u}_{1 t} \boldsymbol{u}_{1 t}^{\prime}=I_{1}$, with $I_{j}$ an $n_{j} \times n_{j}$ identity matrix. Equations (26) and (27) combine to yield the following expression for $\lambda_{1 t}$ :

$$
\lambda_{1 t}=P_{1}^{-1} D_{1}^{1 / 2} \boldsymbol{u}_{1 t}
$$


where $P_{1}^{-1} D_{1}^{1 / 2}$ is lower triangular because it is the product of a lower triangular matrix and a diagonal matrix.

Next, define

$$
\lambda_{3 t}=\theta_{33} \epsilon_{3 t}
$$

and with a sequence of recursive linear projection equations analogous to the sequence used with $\lambda_{1 t}$, obtain

$$
P_{3} \lambda_{3 t}=\rho_{3 t},
$$

where $P_{3}$ is lower triangular with ones along its main diagonal and the covariance matrix for $\rho_{3 t}$ is given by $E \rho_{3 t} \rho_{3 t}^{\prime}=D_{3}$ where $D_{3}$ is a diagonal matrix. Projection errors can then be written as $\boldsymbol{\rho}_{3 t}=D_{3}^{1 / 2} \boldsymbol{u}_{3 t}$, where $\boldsymbol{u}_{3 t}$ has an identity covariance matrix, $E \boldsymbol{u}_{3 t} \boldsymbol{u}_{3 t}^{\prime}=I_{3}$. Hence,

$$
\lambda_{3 t}=P_{3}^{-1} D_{3}^{1 / 2} \boldsymbol{u}_{3 t}
$$

and $P_{3}^{-1} D_{3}^{1 / 2}$ is a lower triangular matrix.

From (25) and (28), obtain $\epsilon_{1 t}=\theta_{11}^{-1} P_{1}^{-1} D_{1}^{1 / 2} \boldsymbol{u}_{1 t}$, and from (29) and (30) solve for $\epsilon_{3 t}=\theta_{33}^{-1} P_{3}^{-1} D_{3}^{1 / 2} \boldsymbol{u}_{3 t}$. Inserting these two expressions into (22) yields

$$
\left[\begin{array}{c}
\Delta \boldsymbol{y}_{1 t} \\
\Delta \boldsymbol{y}_{2 t} \\
\Delta \boldsymbol{y}_{3 t}
\end{array}\right]=\left[\begin{array}{ccc}
\theta_{11}(L) \theta_{11}^{-1} P_{1}^{-1} D_{1}^{1 / 2} & \theta_{12}(L) & \theta_{13}(L) \theta_{33}^{-1} P_{3}^{-1} D_{3}^{1 / 2} \\
\theta_{21}(L) \theta_{11}^{-1} P_{1}^{-1} D_{1}^{1 / 2} & \theta_{22}(L) & \theta_{23}(L) \theta_{33}^{-1} P_{3}^{-1} D_{3}^{1 / 2} \\
\theta_{31}(L) \theta_{11}^{-1} P_{1}^{-1} D_{1}^{1 / 2} & \theta_{32}(L) & \theta_{33}(L) \theta_{33}^{-1} P_{3}^{-1} D_{3}^{1 / 2}
\end{array}\right]\left[\begin{array}{l}
\boldsymbol{u}_{1 t} \\
\boldsymbol{\epsilon}_{2 t} \\
\mathbf{u}_{3 \mathbf{t}}
\end{array}\right] .
$$

The covariance matrix for $\left(\boldsymbol{u}_{1 t}, \boldsymbol{\epsilon}_{2 t}, \boldsymbol{u}_{3 t}\right)$ is by construction the identity matrix. Set $L=1$ in the matrix lag polynomial of equation (31) and, using the restrictions from Assumption 1, obtain the matrix of long-run multipliers:

$$
\left[\begin{array}{ccc}
P_{1}^{-1} D_{1}^{1 / 2} & 0_{12} & 0_{13} \\
\theta_{21} \theta_{11}^{-1} P_{1}^{-1} D_{1}^{1 / 2} & \theta_{22} & 0_{23} \\
\theta_{31} \theta_{11}^{-1} P_{1}^{-1} D_{1}^{1 / 2} & \theta_{32} & P_{3}^{-1} D_{3}^{1 / 2}
\end{array}\right]
$$

Since each block along the main diagonal is a lower triangular matrix and each block above the main diagonal consists of zeros, equation (32) is the matrix of longrun multipliers for a particular long-run recursive model. Consequently, equation (31) is the moving average representation for this recursive model. This result is the key finding of the paper: When an economy has the long-run partially recursive structure given by Assumption 1, a long-run recursive empirical model with the $\left(\boldsymbol{y}_{1}^{\prime}, \boldsymbol{y}_{2}^{\prime}, \boldsymbol{y}_{3}^{\prime}\right)$ ordering will identify structural responses of all variables to $\boldsymbol{\epsilon}_{2 t}$ shocks. The other MARs from this recursive model are linear combinations of structural effects. For example, the dynamic effects obtained for $\boldsymbol{u}_{1 t}$ are a function of the structural responses to $\epsilon_{1 t}$ and the effects for $\boldsymbol{u}_{3 t}$ are a function of the structural responses to $\epsilon_{3 t}$. 


\section{EXAMPLES}

There is much empirical macroeconomic research that identifies VAR models with long-run restrictions. ${ }^{13}$ I will employ the economic structure developed by Amed et al. (1993) to illustrate the identification results from Section 3. One purpose of this section is to provide specific examples of long-run partially recursive structures. A second purpose is to present some of the ways the result from Section 3 can be utilized in empirical research. This discussion illustrates an important point: Economists with different views about which theories best describe economic relationships may nevertheless be able to agree that a particular long-run recursive empirical model is informative about some structural issues.

Amed et al. develop a six-variable open-economy model that includes growth in labor hours for the home country $\left(\Delta n_{h t}\right)$, the home country's output growth $\left(\Delta y_{h t}\right)$, the foreign country's output growth $\left(\Delta y_{f t}\right)$, the difference between private output growth rates between the two countries $\left(\Delta y_{h t}^{p}-\Delta y_{f t}^{p}\right)$, the change in the $\log$ of the terms of trade $\left(\Delta q_{f t}\right)$, and the difference in growth rates of nominal money between the two countries $\left(\Delta m_{h t}-\Delta m_{f t}\right)$. The variables are driven by six structural disturbances. These shocks arise from labor supply in the home country $\left(\tau_{h t}\right)$, worldwide technology $\left(\tau_{t}\right)$, labor supply in the foreign country $\left(\tau_{f t}\right)$, and from cross-country differences in the exogenous shocks to fiscal policy $\left(\eta_{f t}^{*}-\eta_{h t}^{*}\right)$, preferences $\left(\epsilon_{f t}-\epsilon_{h t}\right)$, and the money supply $\left(v_{h t}^{*}-v_{f t}^{*}\right) .{ }^{14}$ Amed et al. assume that the structural shocks are uncorrelated and that the long-run multiplier matrix is completely recursive, writing their model as

$$
\left[\begin{array}{c}
\Delta n_{h t} \\
\Delta y_{h t} \\
\Delta y_{f t} \\
\Delta y_{h t}^{p}-\Delta y_{f t}^{p} \\
\Delta q_{f t} \\
\Delta m_{h t}-\Delta m_{f t}
\end{array}\right]=\left[\begin{array}{cccccc}
\Psi_{11} & 0 & 0 & 0 & 0 & 0 \\
\Psi_{21} & \Psi_{22} & 0 & 0 & 0 & 0 \\
\Psi_{31} & \Psi_{32} & \Psi_{33} & 0 & 0 & 0 \\
\Psi_{41} & \Psi_{42} & \Psi_{43} & \Psi_{44} & 0 & 0 \\
\Psi_{51} & \Psi_{52} & \Psi_{53} & \Psi_{54} & \Psi_{55} & 0 \\
\Psi_{61} & \Psi_{62} & \Psi_{63} & \Psi_{64} & \Psi_{65} & \Psi_{66}
\end{array}\right]\left[\begin{array}{c}
\tau_{h t} \\
\tau_{t} \\
\tau_{f t} \\
\eta_{f t}^{*}-\eta_{h t}^{*} \\
\epsilon_{f t}-\epsilon_{h t} \\
v_{h t}^{*}-v_{f t}^{*}
\end{array}\right]
$$

This representation of a long-run structure comes from first-differencing equation (2) and ignoring the transitory effects of shocks. Their model is motivated by a set of plausible structural assumptions. Amed et al. assume that long-run movements in hours worked are caused exclusively by shocks to a country's labor supply. This restriction can be justified by a vertical labor supply curve. They also assume that a country's output in the long run is driven exclusively by domestic labor supply shocks and worldwide technology shocks. The neoclassical growth model provides this restriction. These assumptions yield the first three equations in (33). ${ }^{15}$ Amed et al. assume fiscal expenditures affect the composition of output between private and government spending but do not affect aggregate output or hours worked in the long run. Their fourth equation allows shocks to home country labor supply, foreign labor supply, technology, and fiscal policy to affect private spending. The fifth 
equation permits the terms of trade to respond in the long run to all of the structural shocks from the first four equations and also to preference shocks. Preference shocks, however, are not allowed to have a long-run effect on private spending, aggregate spending, hours worked, or fiscal policy in the long run. The sixth equation lets the growth of the money supply respond to all shocks in the long run. This specification is motivated by assuming that monetary policy may react to all information that affects macroeconomic outcomes. Money supply shocks have no long-run impact on any variables, except for money, because they assume long-run monetary neutrality. Parameters for this long-run recursive model are estimated by Blanchard and Quah's (1989) technique.

Many alternative structural assumptions to those employed by Amed et al. could have been used. This point does not in any way diminish the value of their research, but instead is a reflection on the field of macroeconomics, which is currently without a unifying paradigm and is undergoing rapid transformation. I will add two more assumptions to the ones chosen by Amed et al. to illustrate the identification results from Section 3:

Assumption A. Permanent technological improvement reduces labor supply in the long run due to a wealth effect;

Assumption B. Fiscal policymakers react to the same information as central bankers.

Assumption A is supported by the observation that per-capita working hours tend to decline as a country becomes more developed. Assumption B is motivated by the fact that monetary and fiscal authorities have similar macroeconomic policy goals and frequently attempt to coordinate policies. If both assumptions are added to Amed et al., the long-run structure becomes

$$
\left[\begin{array}{c}
\Delta n_{h t} \\
\Delta y_{h t} \\
\Delta y_{f t} \\
\Delta y_{h t}^{p}-\Delta y_{f t}^{p} \\
\Delta q_{f t} \\
\Delta m_{h t}-\Delta m_{f t}
\end{array}\right]=\left[\begin{array}{cccccc}
\Psi_{11} & \Psi_{12} & 0 & 0 & 0 & 0 \\
\Psi_{21} & \Psi_{22} & 0 & 0 & 0 & 0 \\
\Psi_{31} & \Psi_{32} & \Psi_{33} & 0 & 0 & 0 \\
\Psi_{41} & \Psi_{42} & \Psi_{43} & \Psi_{44} & \Psi_{45} & \Psi_{46} \\
\Psi_{51} & \Psi_{52} & \Psi_{53} & \Psi_{54} & \Psi_{55} & 0 \\
\Psi_{61} & \Psi_{62} & \Psi_{63} & \Psi_{64} & \Psi_{65} & \Psi_{66}
\end{array}\right]\left[\begin{array}{c}
\tau_{h t} \\
\tau_{t} \\
\tau_{f t} \\
\eta_{f t}^{*}-\eta_{h t}^{*} \\
\epsilon_{f t}-\epsilon_{h t} \\
v_{h t}^{*}-v_{f t}^{*}
\end{array}\right]
$$

The long-run multiplier matrix in (34) has three blocks and is block recursive. The first block contains the first two structural equations, the second block has the foreign output growth equation and the final block consists of the last three equations. The middle block contains a single equation, and since there is only one way to order a single item, this block has a recursive ordering.

Given the structure in (34), consider what happens when Amed et al. estimate a model with the recursive long-run multiplier matrix given by (33). While their empirical model would now be mispecified, the key result from Section 3 shows 
that their long-run recursive ordering will identify structural effects for the foreign labor supply shock. ${ }^{16}$ The MAR for the first two shocks in their recursive ordering would, however, confound the effects of technology and home country labor supply because of Assumption A. Similarly, the effects for the last three shocks in their empirical model will confound the dynamic effects of shocks to fiscal policy, preferences, and money supply because of Assumption B.

Clearly, an appropriate long-run recursive ordering must be employed to identify structural effects. In the general case from Section 3, the middle $n_{2}$ variables must be ordered in a particular sequence. However, the first $n_{1}$ variables can be arbitrarily ordered and so can the last $n_{3}$ variables. For example, given Assumptions A and $\mathrm{B}$, Amed et al. could have identified the effects of foreign labor supply shocks if they had interchanged the first two variables and/or placed the last three variables in some other ordering in a long-run recursive model. In general, there are $n_{1} ! n_{3}$ ! different orderings for the system that will identify structural effects for the $n_{2}$ shocks in the interior block.

Special cases of the general specification of long-run partially recursive structures from Section 3 illustrate how the subset of recursive equations may occur in the first or last block of a system. Suppose that only two sets of block-recursive structural equations exist and the second group consists of recursively ordered equations. This amounts to setting $n_{3}=0$ in the general case from Section 3. Under this assumption, an appropriate long-run recursive ordering will identify the structural MAR for shocks to the last $n_{2}$ equations. The only constraint placed on the initial $n_{1}$ equations is that they are long-run block recursive with respect to the remaining $n_{2}$ equations. For example, suppose that only Assumption $\mathrm{A}$ is added to the structure of Amed et al. In this case, the structure would consist of two blocks where one block contains the first two equations and the other block contains the last four equations. Their recursive ordering would now identify structural effects for the shocks to foreign labor supply, fiscal policy, preferences, and the supply of money. However, when technology shocks have a wealth effect, the first two shocks from their recursive ordering would not identify structural responses.

A second special case is when the block recursive system has two blocks and the first block consists of recursively ordered structural equations. In this case, the second block of equations may be completely unconstrained. This example is handled by setting $n_{1}=0$ in the more general case from Section 3. Hence, the MAR associated with each of the first $n_{2}$ shocks can be identified by an appropriate long-run recursive ordering. For example, if only Assumption B is added to the set of assumptions in Amed et al., the first three equations would form one block and the last three would form another block. In the long run, the first block is block-recursive to the second one and the equations in the first block are equation-by-equation recursive. Therefore, the recursive ordering used by Amed et al. would identify the effects of shocks to home country labor supply, technology, and foreign labor supply. However, the last three shocks from their empirical model would not identify structural responses if the monetary and the fiscal policymakers both respond to all macroeconomic information in the long run. 
It is also worth noting that the basic result from Section 3 is easily extended to a long-run block-recursive system with multiple blocks of long-run recursive equations and possibly more than three blocks. Each block in such a system is easily handled using the methods from Section 3. Hence, long-run recursive orderings consistent with this more complex type of partially recursive structure would be able to identify structural responses for each block of long-run recursive equations.

\section{STRUCTURAL INFERENCE USING MODELS OF COINTEGRATION}

While VAR's with differenced data are quite common in the empirical literature, even more economic research is conducted with models of cointegrated time series. It is, therefore, important to determine if the previous results extend to these models. To address this issue, I use the triangular representation of Phillips (1991), who writes the cointegrated system as a VAR with integrated and stationary variables. Let $s_{t}$ be the linear combinations of time series that are stationary to allow for all cointegrating relationships. ${ }^{17}$ Augment the moving average representation from Section 3 with variables $s_{t}$ and shocks $\boldsymbol{\mu}_{t}$, where the number of additional shocks is equal to the number of stationary variables, to obtain

$$
\left[\begin{array}{c}
\Delta \boldsymbol{y}_{1 t} \\
\Delta \boldsymbol{y}_{2 t} \\
\Delta \boldsymbol{y}_{3 t} \\
\boldsymbol{s}_{t}
\end{array}\right]=\left[\begin{array}{llll}
\theta_{11}(L) & \theta_{12}(L) & \theta_{13}(L) & \theta_{14}(L) \\
\theta_{21}(L) & \theta_{22}(L) & \theta_{23}(L) & \theta_{24}(L) \\
\theta_{31}(L) & \theta_{32}(L) & \theta_{33}(L) & \theta_{34}(L) \\
\theta_{41}(L) & \theta_{42}(L) & \theta_{43}(L) & \theta_{44}(L)
\end{array}\right]\left[\begin{array}{c}
\boldsymbol{\epsilon}_{1 t} \\
\boldsymbol{\epsilon}_{2 t} \\
\boldsymbol{\epsilon}_{3 t} \\
\boldsymbol{\mu}_{t}
\end{array}\right]
$$

Assume that the $\boldsymbol{\mu}_{t}$ shocks are transitory shocks in the sense that they do not have a permanent effect on any variables. This is equivalent to having the number of independent permanent structural shocks match the number of differenced series in (35). The common stochastic trends representation for cointegrated systems developed by Stock and Watson (1988) justifies this framework. Transitory structural shocks are assumed to be uncorrelated with the permanent shocks. This assumption is critical for identifying the effects of permanent shocks. The transitory shocks may, however, be correlated with one another without posing any difficulties.

Since transitory shocks, by definition, do not have a permanent effect on integrated variables, the following restrictions must hold: $\theta_{14}(1)=0_{14}, \theta_{24}(1)=0_{24}$, and $\theta_{34}(1)=0_{34}$. However, $\theta_{41}(1), \theta_{42}(1), \theta_{43}(1)$, and $\theta_{44}(1)$ parameters are unconstrained. These parameters do not reflect permanent effects because they pertain to stationary variables, which cannot experience a permanent response to any kind of shock.

Assume an arbitrary set of identification restrictions, ${ }^{18} \boldsymbol{\mu}_{t}=\Omega \boldsymbol{u}_{4 t}$, which map the transitory structural shocks into a set of orthogonal shocks $u_{4 t}$. If the structure is long-run partially recursive of the form given in Assumption 1, then the same method from Section 3 can be used here to map the long-run partially recursive structure from equation (35) into the long-run recursive model 
$\left[\begin{array}{c}\Delta \boldsymbol{y}_{1 t} \\ \boldsymbol{\Delta}_{2 t} \\ \Delta \boldsymbol{y}_{3 t} \\ \boldsymbol{s}_{t}\end{array}\right]=\left[\begin{array}{llll}\theta_{11}(L) \theta_{11}^{-1} P_{1}^{-1} D_{1}^{1 / 2} & \theta_{12}(L) & \theta_{13}(L) \theta_{33}^{-1} P_{3}^{-1} D_{3}^{1 / 2} & \theta_{14}(L) \Omega \\ \theta_{21}(L) \theta_{11}^{-1} P_{1}^{-1} D_{1}^{1 / 2} & \theta_{22}(L) & \theta_{23}(L) \theta_{33}^{-1} P_{3}^{-1} D_{3}^{1 / 2} & \theta_{24}(L) \Omega \\ \theta_{31}(L) \theta_{11}^{-1} P_{1}^{-1} D_{1}^{1 / 2} & \theta_{32}(L) & \theta_{33}(L) \theta_{33}^{-1} P_{3}^{-1} D_{3}^{1 / 2} & \theta_{34}(L) \Omega \\ \theta_{41}(L) \theta_{11}^{-1} P_{1}^{-1} D_{1}^{1 / 2} & \theta_{42}(L) & \theta_{43}(L) \theta_{33}^{-1} P_{3}^{-1} D_{3}^{1 / 2} & \theta_{44}(L) \Omega\end{array}\right]\left[\begin{array}{c}\boldsymbol{u}_{1 t} \\ \boldsymbol{\epsilon}_{2 t} \\ \boldsymbol{u}_{3 t} \\ \boldsymbol{u}_{4 t}\end{array}\right]$

Setting $L=1$ in (36) shows that the long-run multiplier matrix for the effects of permanent shocks $\left(\boldsymbol{u}_{1 t}, \boldsymbol{\epsilon}_{2 t}, \boldsymbol{u}_{3 t}\right)$ on the integrated variables $\left(\boldsymbol{y}_{1 t}^{\prime}, \boldsymbol{y}_{2 t}^{\prime}, \boldsymbol{y}_{3 t}^{\prime}\right)$ is given by the lower triangular matrix in equation (32). Since (36) is the MAR obtained from this particular ordering of long-run multipliers, the effects of $\epsilon_{2 t}$ are identified by this long-run recursive empirical model. In the Appendix, I show how the same result obtains if the cointegrated system is specified using a vector error correction model. Hence, the analysis of long-run partially recursive structures in Sections 3 and 4 extends to models of cointegration.

\section{CONCLUDING COMMENTS}

This paper proves that a long-run partially recursive structure is sufficient for longrun recursive orderings to identify some structural effects, as long as the ordering is consistent with the underlying structure. One can also show that two-block versions of the long-run partially recursive structure are necessary for the initial block or the final block of shocks from a long-run recursive ordering to yield structural responses. These results follow from a simple extension of necessary conditions ${ }^{19}$ from Section 5 of Keating (1996): Assume the variables are integrated and replace the short-run structural parameter matrix and the short-run identification restrictions with the long-run partially recursive structural parameter matrix and the long-run recursive identification restrictions, respectively. ${ }^{20}$

A key implication of this paper is that long-run recursive empirical models yield structural responses for a large class of economic structures, not simply for the set of structures that are fully recursive in the long run. The most natural application of the result would be if theory provides a particular long-run partially recursive structure. Then it is clear that an appropriate long-run recursive empirical model can be used to address specific economic questions. A second application could be for economists to reexamine existing empirical studies that have used long-run recursive models. It might be possible to reinterpret these studies in the context of different theoretical assumptions. If an alternative theory yields a long-run partially recursive system and the empirical study happens to use a long-run recursive model consistent with that theory, then the empirical model will be able to address certain questions that pertain to that alternative theory. Researchers who want to attach structural interpretations to empirical models of integrated or cointegrated time series may find these applications useful and may develop new ways of using the results developed in this paper. 


\section{NOTES}

1. See Strotz and Wold (1960) for classic references to this literature.

2. Note, however, that economic theory often generates plausible long-run recursive structures. For example, following Blanchard and Quah (1989), much of this literature uses the assumption that aggregate demand has a long-run neutral effect on output, and this assumption typically yields a longrun recursive economic structure. Long-run recursive structures also have been developed by Bullard and Keating (1995) and Roberts (1993) to address the superneutrality of money.

3. Their results are derived for long-run structures that are not necessarily recursive. In fact, some of their results also apply to short-run structures, although Faust and Leeper do not emphasize this.

4. A single set of identification restrictions is typically used in most work with VAR's, and there always seem to be some economists who question the robustness of the results to alternative identification assumptions. King and Watson (1997) present an interesting method of examining the results from virtually all possible identification restrictions for a bivariate VAR. In the bivariate case, the model is identified by restricting a single parameter, assuming that structural shocks are uncorrelated, and robustness can be addressed by graphing the way certain features of the identified system change as this parameter is varied. However, it is not clear how to extend their graphical method to larger VAR systems because these models are identified by choosing values for more than one parameter. Of course, robustness is a general concern for all empirical analysis, not a specific problem with VAR's.

5. The structure in Shapiro and Watson (1988) has a "lower block triangular" matrix of long-run multipliers. The results shown here hold for their system and for more complicated examples from the class of long-run partially recursive structures.

6. Of course, the empirical model would need to account for any deterministic features in the data.

7. Equation (2) is constructed by integrating equation (1).

8. Lippi and Reichlin (1993) and Blanchard and Quah (1993) present alternative views on invertibility. Noninvertible structures can be constructed from the VAR's fundamental representation.

9. Equation (4) assumes $\ell$ lags will adequately represent the infinite-order structural MAR in (1).

10. Hamilton $(1994$, p. 91$)$ proves that the triangular factor is unique.

11. Bernanke (1986), Blanchard and Watson (1986), and Sims (1986) are foundational works on structural VAR identification with restrictions on $\theta(0)$. Keating (1990) shows that the commonly used exclusion restrictions in this approach may be invalid if agents are forward looking and have rational expectations. West (1990) also uses rational expectations in a structural VAR. Gali (1992) combines short-run and long-run restrictions.

12. Technically speaking, if the covariance matrix of structural shocks is block diagonal and if each shock in $\epsilon_{2 t}$ is uncorrelated with every other shock, then the results will still go through. This assumption allows shocks in the first block to be correlated with one another and shocks in the third block to be correlated with one another, but no correlation between shocks from different blocks.

13. See Amed et al. (1993), Gamber and Joutz (1993), Lastrapes and Selgin (1994), Bullard and Keating (1995), Faust and Leeper (1997), King and Watson (1997), Keating and Nye (1998), and the references in these papers to additional research.

14. Asterisks are used to indicate that some structural parameters multiply monetary and fiscal shocks in their model. I use this simplified notation because these parameters are irrelevant for my purposes.

15. These assumptions imply $\boldsymbol{\Psi}_{31}=0$, but since Amed et al. ignore this restriction, I will also ignore overidentification issues.

16. The fact that $\Psi_{56}=0$ in (34) does not imply that additional structural responses can be obtained from this or any other recursive ordering.

17. A particularly simple cointegrating vector obtains for any stationary variable.

18. The restrictions associated with $\boldsymbol{u}_{4 t}$ would most likely come from short-run identification assumptions. 
19. However, the sufficient conditions in this paper do not obtain from a simple extension of Keating's (1996) results.

20. Equation (20) from this paper can be used to justify these substitutions.

\section{REFERENCES}

Amed, S., B.W. Ickes, P. Wang, \& B.S. Yoo (1993) International business cycles. American Economic Review 83, 335-359.

Bernanke, B. S. (1986) Alternative explanations of the money-income correlation. Carnegie-Rochester Conference Series on Public Policy 25, 49-100.

Beveridge, S. \& C.R. Nelson (1981) A new approach to decomposition of economic time series into permanent and transitory components with particular attention to measurement of the "business cycle." Journal of Monetary Economics 7, 151-174.

Blanchard, O.J. \& D. Quah (1989) The dynamic effects of aggregate demand and supply disturbances. American Economic Review 79, 655-673.

Blanchard, O.J. \& Danny Quah (1993) The dynamic effects of aggregate demand and supply disturbances: Reply. American Economic Review 83, 653-658.

Blanchard, O.J. \& M.W. Watson (1986) Are business cycles all alike? In R. J. Gordon, (ed.), The American Business Cycle: Continuity and Change, pp. 123-156. Chicago: University of Chicago Press.

Ballard, J. \& J.W. Keating (1995) The long-run relationship between inflation and output in postwar economies. Journal of Monetary Economics 36, 477-496.

Cooley, T.A. \& S.F. LeRoy (1985) Atheoretical macroeconomics: A critique. Journal of Monetary Economics 16, 283-308.

Engle, R.F. \& C.W.J. Granger (1987) Cointegration and error correction: Representation, estimation and testing. Econometrica 55, 251-276.

Faust, J. \& E.M. Leeper (1997) When do long-run identifying restrictions give reliable results? Journal of Business and Economic Statistics 15, 345-353.

Gali, J. (1992) How well does the IS-LM model fit postwar U.S. data? Quarterly Journal of Economics 57, 709-738.

Gamber, E.N. \& F.L. Joutz (1993) The dynamic effects of aggregate demand and supply: Comment. American Economic Review 83, 1387-1393.

Hamilton, J.D. (1994) Time Series Analysis. Princeton, NJ: Princeton University Press.

Keating, J.W. (1990) Identifying VAR models under rational expectations. Journal of Monetary Economics 25, 453-476.

Keating, J.W. (1996) Structural information in recursive VAR orderings. Journal of Economic Dynamics and Control 20, 1557-1580.

Keating, J.W. \& J.V. Nye (1998) Permanent and transitory shocks in real output: Estimates from nineteenth century and postwar economies. Journal of Money, Credit and Banking 30, 231-251.

King, R.G., C.I. Plosser, J.H. Stock, \& M.W. Watson (1991) Stochastic trends and economic fluctuations. American Economic Review 81, 819-840.

King, R.G. \& M.W. Watson (1997) Testing long-run neutrality. Economic Quarterly, Federal Reserve Bank of Richmond 83/3(Summer), 69-101.

Lastrapes, W.D. \& G.A. Selgin (1994) Buffer stock money: Interpreting short-run dynamics using long-run restrictions. Journal of Money, Credit, and Banking 26, 34-54.

Lippi, M. \& L. Reichlin (1993) The dynamic effects of aggregate demand and supply disturbances: Comment. American Economic Review 83, 644-652.

Pagan, A.R. \& J.C. Robertson (1998) Structural models of the liquidity effect. Review of Economics and Statistics 80, 202-217.

Phillips, P.C.B. (1991) Optimal inference in cointegrated systems. Econometrica 59, 283-306.

Roberts, J. (1993) The sources of business cycles: A Monetarist interpretation. International Economic Review 34, 923-934. 
Sarte, P.D.G. (1997) On the identification of structural vector autoregressions. Economic Quarterly, Federal Reserve Bank of Richmond 83/3(Summer), 45-67.

Shapiro, M.D. \& M.W. Watson (1988) Sources of business cycle fluctuations. NBER Macroeconomics Annual 3, 111-148.

Sims, C.A. (1980) Macroeconomics and reality. Econometrica 48, 1-48.

Sims, C.A. (1986) Are forecasting models usable for policy analysis? Quarterly Review, Federal Reserve Bank of Minneapolis 10, 2-16.

Stock, J.H. \& M.W. Watson (1988) Testing for common trends. Journal of the American Statistical Association 83, 1097-1107.

Strotz, R.H. \& H.O.A. Wold (1960) Recursive vs. nonrecursive systems: An attempt at synthesis. Econometrica 28, 417-427.

West, K.D. (1990) The sources of fluctuations in aggregate inventories and GNP. Quarterly Journal of Economics 55, 939-971.

\section{APPENDIX: STRUCTURAL INFERENCE USING VECTOR ERROR CORRECTIONS MODELS}

The vector error corrections model (VECM) of Engle and Granger (1987) is probably the most popular model for cointegrated time series. The order of integration is arbitrary but, for convenience, assume $\boldsymbol{X}_{\boldsymbol{t}}$ is a vector of $m$ variables that are integrated of order 1 . Let $\beta^{\prime} \boldsymbol{X}_{t}$ be the stationary linear combinations of $\boldsymbol{X}$. If there are $m-n$ cointegrating vectors, then $\beta$ is an $m \times(m-n)$ matrix, where $0<n<m$. The number of transitory shocks is equal to the number of cointegrating vectors, and the number of stochastic trends in the system must equal $n$ based on Stock and Watson's (1988) common trends representation. In a VECM, dependent variables are differenced enough times to become stationary and the set of regressors includes lags of these differenced variables along with the stationary linear combinations of undifferenced variables.

King et al. (1991) develop a method to identify permanent shocks in a VECM based on a recursive ordering of permanent components. They write the moving average representation for a cointegrated system as $\Delta \boldsymbol{X}_{t}=\Gamma(L) \boldsymbol{\eta}_{t}$, where $\Gamma(L)$ is obtained by inverting the VECM and

$$
\boldsymbol{\eta}_{t}=\left[\begin{array}{c}
\boldsymbol{\eta}_{t}^{P} \\
\boldsymbol{\eta}_{t}^{T}
\end{array}\right],
$$

where $\boldsymbol{\eta}^{P}$ are the permanent shocks and $\boldsymbol{\eta}^{T}$ are the transitory shocks. Permanent shocks are assumed to be uncorrelated with one another. Although the transitory shocks are not necessarily identified, the assumption that transitory shocks are uncorrelated with permanent shocks is crucial for identifying the permanent shocks. The long-run effects of permanent shocks are constrained by the cointegrating relationships and by the economist's interpretation of the sources of the permanent components. King et al. show that the long-run multipliers can be written as 


$$
\Gamma(1)=\left[\tilde{A} \pi, \quad 0_{m, m-n}\right],
$$

where $\tilde{A}$ is an $m \times n$ matrix of coefficients derived from the parameters in $\beta$ and $\pi$ is a full-rank $n \times n$ matrix. The moving average representation also can be written as $\Delta \boldsymbol{X}_{t}=\Gamma(1) \boldsymbol{\eta}_{t}+\Gamma^{*}(L) \Delta \boldsymbol{\eta}_{t}$ to separate permanent effects from transitory dynamics. From the previous expressions, the permanent effects are given by $\Gamma(1) \boldsymbol{\eta}_{t}=\tilde{A} \pi \boldsymbol{\eta}_{t}^{P}$. King et al. assume that $\pi$ is lower triangular, and show how to obtain $\pi$ from the Cholesky decomposition of a matrix constructed from parameters of the VECM. The permanent components from their recursive model can be written as $\boldsymbol{e}_{t}^{P}=\pi \boldsymbol{\eta}_{t}^{P}$, where $\boldsymbol{e}_{t}^{P}$ represents permanent movements in particular variables. King et al. find three cointegrating vectors in their sixvariable model, which implies that the system has three independent permanent shocks. They identify the permanent components with real output, inflation, and the real interest rate. Estimation of $\pi$ allows them to decompose the permanent components for these three variables into three orthogonal permanent shocks $\boldsymbol{\eta}^{P}$. If the economic structure happens to be long-run recursive and the correct ordering of variables is used, then clearly each permanent shock will identify structural effects.

In the general case, suppose that the permanent movements in variables are related to structural disturbances by the following system of equations: $\boldsymbol{e}_{t}^{P}=\theta(1) \boldsymbol{\epsilon}_{t}$. Suppose that this structural system is partially recursive in the long run with the particular form given in Assumption 1, implying that the structure can be written as

$$
\left[\begin{array}{l}
\boldsymbol{e}_{1 t}^{P} \\
\boldsymbol{e}_{2 t}^{P} \\
\boldsymbol{e}_{3 t}^{P}
\end{array}\right]=\left[\begin{array}{lll}
\theta_{11} & 0_{12} & 0_{13} \\
\theta_{21} & \theta_{22} & 0_{23} \\
\theta_{31} & \theta_{32} & \theta_{33}
\end{array}\right]\left[\begin{array}{l}
\boldsymbol{\epsilon}_{1 t} \\
\boldsymbol{\epsilon}_{2 t} \\
\boldsymbol{\epsilon}_{3 t}
\end{array}\right]
$$

with $\theta_{22}$ a lower triangular matrix. The long-run recursive empirical model can be written as

$$
\left[\begin{array}{c}
\boldsymbol{e}_{1 t}^{P} \\
\boldsymbol{e}_{2 t}^{P} \\
\boldsymbol{e}_{3 t}^{P}
\end{array}\right]=\left[\begin{array}{lll}
\pi_{11} & 0_{12} & 0_{13} \\
\pi_{21} & \pi_{22} & 0_{23} \\
\pi_{31} & \pi_{32} & \pi_{33}
\end{array}\right]\left[\begin{array}{l}
\boldsymbol{\eta}_{1 t}^{P} \\
\boldsymbol{\eta}_{2 t}^{P} \\
\boldsymbol{\eta}_{3 t}^{P}
\end{array}\right]
$$

to conform with these structural blocks. Using precisely the same transformations found in Section 3, it is easy to map the long-run partially recursive structure into this recursive model, and find that $\pi_{22}=\theta_{22}, \pi_{32}=\theta_{32}$ and $\boldsymbol{\eta}_{2 t}^{P}=\boldsymbol{\epsilon}_{2 t}$. Then it is straightforward (using essentially the analysis from Section 3 ) to show that, given the structure in Assumption 1, $\boldsymbol{\eta}_{2 t}^{P}$ will identify the dynamic response of each variable to $\epsilon_{2 t}$. 